\title{
A PRELIMINARY ASSESSMENT OF SLENDERNESS AND OVERSTRENGTH HOMOGENEITY CRITERIA USED IN THE DESIGN OF CONCENTRICALLY BRACED STEEL FRAMES IN MODERATE SEISMICITY
}

\author{
Alper Kanyilmaz ${ }^{1}$, Carlo A. Castiglioni ${ }^{2}$, Hervé Degèe ${ }^{3}$, Pierre-Olivier Martin ${ }^{4}$ \\ 1,2 Department of Architecture, Built Environment and Construction Engineering \\ Politecnico di Milano \\ Via Ponzio 31, 20133 Milano, Italy \\ alper.kanyilmaz@polimi.it, carlo.castiglioni@polimi.it \\ ${ }^{3}$ Hasselt University, Belgium \\ herve.degee@uhasselt.be \\ ${ }^{4}$ CTICM, France \\ pmartin@cticm.com
}

Keywords: Moderate seismicity; steel structures; concentrically braced frames, slenderness, overstrength homogeneity

\begin{abstract}
Concentrically braced frames (CBF) represent a very effective structural form against horizontal loading. They provide high lateral resistance at the same time limiting lateral displacements. Thanks to their simpler connection details and smaller cross sections, they are economic alternatives to the costly moment resisting frames. Nevertheless, concerning the seismic design, current Eurocode 8 provisions require a quite high level of complexity for the dissipative design of $C B F$ structures. The global aim of this research is to find an optimal balance between safety and economy for the design of CBF structures, located in low-tomoderate seismic regions. New design rules will be proposed which will have less stringent local ductility and structural homogeneity requirements than current medium ductility class (DCM), and provide necessary safety level limiting the complexity and costs associated with anti-seismic design. This paper presents the results of the preliminary numerical analysis that has been realized thanks to the research fund received from European commission with the contract MEAKADO RFSR-CT-2013-00022.
\end{abstract}




\section{INTRODUCTION}

The general philosophy of the most recent seismic design codes is mainly based on the assumption of providing the structure with global and fully developed plasticity, through the application of capacity design principles. However, the application of these principles in lowto-moderate seismic zones often leads to complex solutions, which are difficult to implement and usually results in significant increases in the construction costs. For this reason, building designers often neglect the capacity design principles, and apply low ductility design (DCL with $\mathrm{q}=1.5$ ) in low-to-moderate seismic zones [1]. Given the unpredictable behaviour of low or moderate seismic actions, this approach does not always provide safe and economic solutions.

The ductility classifications are based on the rules developed in the $80 \mathrm{~s}$ which led to the first official publication of Eurocode 8 (May 1988 edition). The behavior factors defined in this edition remained nearly unchanged since then, only some restrictions between moderate ductility design (DCM) and high ductility design (DCH) were introduced. A common aim of almost all research projects carried out since then was to verify and validate the behaviour factors as assessed in 1988.

Regarding the strict application of current Eurocode 8 [2] for regions presenting a low-tomoderate seismicity level (Belgium, Spain, Germany, France, Austria, Portugal and some parts of Italy) the following observations can be made:

- The use of DCL principle is only recommended for low seismicity regions in which the design ground acceleration on type A ground, $a_{\mathrm{g}}$, is not greater than $0.08 \mathrm{~g}$, or those where the product $a_{\mathrm{g}} . S$ is not greater than $0.1 \mathrm{~g}$. National authorities in Europe tend to increase PGA values beyond 0.1g (Latest example, France).

- The use of DCM principle requires the application of all design rules prescribed to provide ductility to the structure (same rules as $\mathrm{DCH}$ principle).

In this case, designers have two options in low-to-moderate seismicity regions:

i) Design according to DCM, using high q factors to reduce the design forces. In this case, significant local ductility and structural homogeneity requirements have to be fulfilled. Furthermore, the over assessed behaviour factor needs to be corrected by the global over-strength factor $\Omega$ which leads to even more complex design.

ii) Design according to DCL, which means design for unreduced seismic actions, without any guarantee and control of ductility and thus a very limited reliability level. Although DCL method is not recommended, it is allowed by Eurocode for moderate seismic zones.

The first approach, if applied accurately, leads to reliable and safe structures, but the cost may be too high since no distinction is made with respect to the design of structures to be built in high seismic zones. The second approach is clearly unreliable, because not even a minimum effort is paid for the control of ductility. It can be also expensive because of limited values allowed for the behavior factor.

Research in earthquake engineering has been essentially realized for applications in regions exhibiting a very significant seismicity level, with the aim of preventing brittle collapses, and maximizing the capacities in terms of energy dissipation. There are very few research projects, which investigate the problem in moderate seismic zones. Some of them provide new approaches for reinforced concrete structures, some suggest considering the contribution of gravity systems to the lateral system, and some gives design tips using the available standards (mostly US standards) to come up with safer and economic design. Pinto (2000) [3], emphasizes the fact that seismic design in low/moderate hazard regions cannot have a single, rational solution applicable to all cases. Application of seismic design principles requires a compromise to be reached based on each specific situation according to physical, quantitative knowledge of 
the hazard and awareness of it from the public, capacity on the part of the authorities to regulate the construction process and to impose the use of anti-seismic standards, and availability of the resources necessary for ensuring protection against different levels of damage. Dominic et al. (2006) [4], highlight the fact that in United States, building designers and constructors based in moderate seismic areas do not have extensive experience with earthquake-resistant construction. The consequence of misusing complicated seismic provisions could result in unsafe and unnecessarily costly buildings. Therefore the authors provide design tips which include determining site class and seismic design category, selecting a steel seismic-force-resisting system, and applying detailing requirements according to the American standards. Hines and Fahnestock (2010) [5], point out that from low seismic design to high seismic, the costs of design and fabrication and probability of making mistakes in both processes increase. They suggest that having a flexible reserve system in moderate seismic areas can provide a moderate ductility, which can improve the seismic behavior of steel structures in these zones while keeping the design as simple as possible. Their proposals are addressed to the designers who use American Standards. Hines et.al (2009) [6], propose an independent seismic design philosophy for low-ductility structures in moderate seismic regions. They use the concepts of reserve capacity and elastic flexibility, which should complement better-established concepts of strength, ductility and capacity design. The paper focuses specifically on the concept of reserve capacity and its ability to improve collapse performance for chevron braced steel frames not specifically detailed for seismic resistance. They keep the behavior factor small as possible $(\mathrm{R}=3$, according to American Standards) and try to exploit the energy dissipation characteristics of the gravity system which serves as reserve capacity, although this is not permitted by the standards. They also make an interesting comparison between a low ductility system with $\mathrm{R}=3$ and high ductility system with $\mathrm{R}=5$ in terms of economy. They find that the system with high ductility design costs $82 \%$ more. Although an $R=3$ design may not safely conform to the seismic design philosophy for moderate seismic regions, this comparison shows whether increased ductility is really the most economical approach to ensuring collapse resistance in moderate seismic regions. Elghazouli (2008) [7] explains the necessity of using over-strength homogeneity rule which is not considered explicitly in other codes, however he also accepts that this requirement in isolation cannot eliminate the problem even when the $25 \%$ limit is reduced. Moreover, he also mentions that this rule imposes additional design effort and practical difficulties in the selection of brace sizes. He believes that relaxing this limit can be possible by increasing the continuity and stiffness of the columns. Gioncu And Mazzolani (2014) [8] in their new book "Seismic Design of Steel Structures", dedicated an entire section "Low-toModerate Seismic regions", which represents a large area of the world, under the chapter of "Challenges in Seismic Design". They explain the main issues regarding the seismic design in these areas, which include main characteristics of low-to-moderate ground motions and structural design problems in these regions. They underline the fact that seismic risk in regions with low-to-moderate seismicity is an important question without clear answers. Murty and Malik (2008) [9] raises the challenges in the current design practice in the large low-tomoderate seismic regions of India, where over the last decade; there has been a sudden surge in the construction activity. Among their proposals for the future are the new design strategies suited for low-to-medium seismic regions, in particular implementing an awareness campaign for all stakeholders especially in low-to-moderate seismic regions, and developing and updating seismic design provisions towards improving earthquake safety, especially for the low-tomoderate seismic regions. The paper published By Elghazouli (2003) [10] deals with the main behavioral issues of seismic design of concentrically braced frames. Eurocode 8 recommendations on ductility demand and inelastic distribution are examined through dynamic nonlinear analysis on idealized frame configurations. Finally, several code modifications are 
proposed in order to ease causes of undesirable performance and to facilitate a rational implementation of capacity design concepts.

An extensive literature review makes it evident that low-to-medium seismicity is currently a fresh topic in the research field, with many promises but very few accomplishments. Researches all around the world accept the problem, and are trying to develop solutions. However, so far the achievements are quite limited. Most of the research in earthquake engineering focuses on applications in regions exhibiting a very significant seismicity level, with the aim of preventing brittle collapses and maximizing the capacities in terms of energy dissipation. Among the few researches on low-to-medium seismicity, some of them are providing new approaches for reinforced concrete structures, some suggest to consider the contribution of gravity systems to the lateral system, and some gives design tips using the available standards (mostly US standards) to come up with safer and more economic design. In Europe, only a few researches raised the moderate seismic design necessity. There is an ongoing European RFCS research project MEAKADO, which is investigating the moderate seismic design rules for concentrically braced frames (CBF) and moment resisting frames (MRF), as being the most relevant typologies in the European construction market.

\section{METHOD AND ADVANCEMENTS}

According to current version of Eurocode 8, a considerable behaviour factor for CBF structures bigger than 1,5 or 2 can only be taken into account if stringent rules on the bracing slenderness and on the over-strength homogeneity are fulfilled. In practice, these requirements are actually very difficult, and sometimes even impossible to meet. In this research, alternative design solutions are being developed with relaxed over-strength homogeneity and slenderness criteria.

By means of nonlinear transient dynamic analysis under several accelerograms, performance of case studies designed according to Eurocodes, and with relaxed capacity design rules, are evaluated. These case studies are designed by project partner CTICM (within Task 3.2 of MEAKADO project). The types of bracing considered include (figure 1):

- $\mathrm{X}$ bracings where diagonals in tension and diagonals in compression are in the same span;

- $\mathrm{N}$ bracings where diagonals in tension and diagonals in compression are in two different spans;

- V bracings.
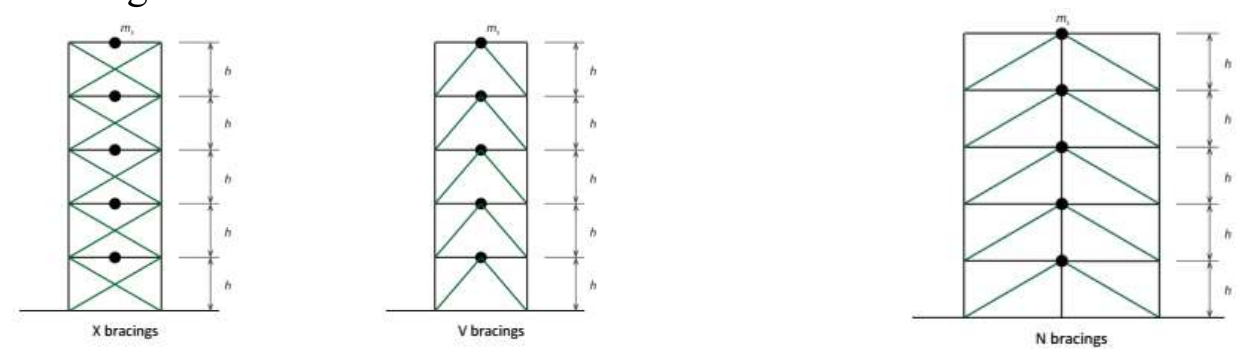

Figure 1 Frame types used in case studies by CTICM

First of all, influence of different modeling parameters has been studied on the case study frames. The parameters studied are beam end boundary conditions, initial imperfection, and presence of rigid links, damping values, and load distribution schemes. The results obtained from the numerical analysis have been compared with those provided by CTICM, which were 
obtained from linear static analysis with response spectrum. Following observations can be made:

i) Effect of initial imperfection amount: In general, with smaller imperfections, maximum compression forces achieved in bracings are higher, the maximum displacements achieved at each floor level is smaller (the rate of difference changes randomly at each floor), maximum base shear is not very different for $4 \mathrm{~N}-\mathrm{CHS}$ frame, but can be quite higher in case of 4V-UPE frame. All the noted differences (compression forces in bracings, floor displacements and maximum base shear) is much higher when the 4V-UPE frame is modelled with simpler boundary conditions (beam-ends and column bases hinged, no rigid links at the frame).

ii) Presence of Rigid links at the connections: In general, presence of rigid links at connections increase the lateral stiffness of the structure, decreases floor displacements, increases base shears and maximum compression forces achieved in bracings. In case of $4 \mathrm{~V}$-UPE frame, the differences observed in floor displacements are higher when the frame is modelled with simpler boundary conditions (beam ends and column bases hinged).

iii) Damping value: With more damping in numerical analysis, floor displacements, base shear and maximum forces obtained in the bracings are lower.

iv) Load distribution on beams: Distribution of gravity loads either on beams, or concentrated on columns directly, do not have an important effect for 4N-CHS frame. $4 \mathrm{~V}$-UPE frame had differences in the order of $10 \%$, in terms of displacements and base shears depending on how the gravity loads are imposed (concentrated on columns, or distributed on beams)

v) Frame effect: In general, important differences are observed when these two different approaches are adapted to simulate the boundary conditions: i) basic boundary conditions with beam ends, column bases are hinged and no rigid links at connections and ii) boundary conditions with beam ends and column bases are fixed, and rigid links are used to simulate gusset plates and offsets. The comparisons between numerical models and experiments showed that latter boundary conditions can be more realistic. This is probably because with basic boundary conditions, the "frame effect" provided by CBF structure is neglected, while in reality it has a quite important participation in the overall behaviour. Also a linear static analysis on $4 \mathrm{~N}-$ CHS frame showed that, contribution of "frame effect" on the overall stiffness can be in the order of $15 \%$. Studies on this issue are still underway, and will be validated by experimental work during the research project.

In the FE model, 3 sets of artificial accelerograms have been considered. Input for the target response spectrum can be seen in Table 1.

\begin{tabular}{|l|l|}
\hline PGA (for soil type A) & $0.15 \mathrm{~g}$ \\
\hline Soil Type for target response spectrum & B \\
\hline Spectrum Type & 2 \\
\hline
\end{tabular}

Table 1 Input for target response spectrum

Following assumptions have been made during FE modeling:

i. Columns are continuous along the height

ii. Bracings are pinned at their ends. Beam ends and column bases are pinned.

iii. Rigid links are used at the end of bracings to take into account gusset plates (with $34 \mathrm{~cm}$ length, accounting for $\mathrm{L}_{\mathrm{cr}}=0.9 \mathrm{~L}$ design assumption) 
iv. Lumped dynamic masses are used in the middle of beam elements

v. Material and geometrical nonlinearities have been taken into account

vi. Bracings are modelled with fiber-based nonlinear elements with distributed plasticity. Numerical validation studies have been performed on the use of fiber-based nonlinear beam elements with distributed plasticity approach, which are presented in separate articles [11, 12].

vii. Column and beam elements are modelled as elastic

viii. Initial imperfections of L/250 have been used for CHS bracing elements.

ix. The beam cross sections are divided into 16 fibers as circumferential divisions, each fiber is monitored independently with respect to its position on the stress-strain table and the integration of all the fibers over the length, determines effective beam properties. 5 integration points have been used for every section.

x. Bracing and beam elements are subdivided into 20, columns are subdivided into 8, beams are subdivided into 16 elements (meshes) along their length

xi. The analysis is made in three steps: First, a linear static analysis is performed with a displacement applied in the middle of the bracing which is equal to $\mathrm{L} / 250$, then based on the deformed shape of this model, nonlinear static analysis is performed only under vertical loads, and finally nonlinear transient dynamic analysis is performed starting from the last step of the nonlinear static analysis.

xii. A viscous damping is usually adopted for nonlinear dynamic analyses, which require a direct integration of the uncoupled motion equations. A particular model of viscous damping, which finds applications in large commercial softwares such as Straus7, is the Rayleigh damping. In the Rayleigh damping model, damping matrix $\mathrm{c}$ is a linear combination of the mass matrix $\mathrm{m}$ and stiffness matrix $\mathrm{k}$ :

$$
\text { a. } \boldsymbol{c}=a_{0} \boldsymbol{m}+a_{1} \boldsymbol{k}
$$

b. Where $\mathrm{a}_{0}[1 / \mathrm{s}]$ and $\mathrm{a}_{1}[\mathrm{~s}]$ are proportionality constants, which depend on the first two natural frequencies of the structure. In this equation, the stiffness matrix is updated at each step, keeping constant $\mathrm{a}_{0}$ and $\mathrm{a}_{1}$.

c. In the models, $2 \%$ Rayleigh damping has been used.

xiii. S275 steel has been used with elasto-plastic material with kinematic strain hardening of 1\% (ref. eurocode 3)

\begin{tabular}{|r|r|}
\hline Strain & Stress (MPa) \\
\hline 0 & 0 \\
\hline 0 & 275 \\
\hline 0,075 & 430 \\
\hline 0,15 & 430 \\
\hline
\end{tabular}

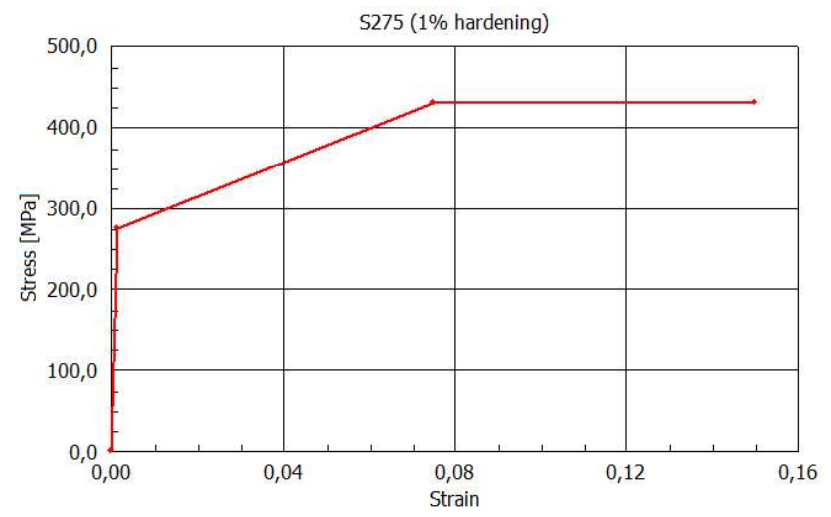

Table 2 Material properties S275

xiv. Design aacceleration inputs have been multiplied by 1.18 to take into account the effect of accidental torsion 
The objective of this analysis is to assess the impact of the relaxation of slenderness and overstrength limits of Eurocode 8, on the seismic performance of CBF buildings under moderate seismic actions.

Overstrength criterion for CBF structures is defined as follows in EC8:

$$
\Omega_{\mathrm{i}} \leq 1,25 \Omega
$$

For buildings with more than two levels, slenderness criterion for CBF structures is defined as follows in EC8:

$$
\begin{gathered}
\bar{\lambda} \leq 2,0 \text { for } \mathrm{N} \text { and } \mathrm{V} \text { bracings } \\
1,3 \leq \bar{\lambda} \leq 2,0 \text { for } \mathrm{X} \text { bracings }
\end{gathered}
$$

The target of the parametric study is to assess the effect of relaxed criteria on the behaviour of steel structures in moderate seismicity areas. This study proposes some case studies to be considered.

The two following criteria will be assessed, first alone and secondly coupled:

$$
\begin{gathered}
\text { Overstrength criterion: } \Omega_{\mathrm{i}} \leq 1,50 \Omega \\
\text { Slenderness criterion: } \quad \bar{\lambda} \leq 2,5
\end{gathered}
$$

The review of the regulatory limits proposal is shown in Table 2:

\begin{tabular}{|c|c|}
\hline EN1998-1 rules & $\Omega_{\max }<\mathbf{1 , 2 5} \Omega_{\min }$ \\
& $\mathbf{1 , 3}<\lambda<\mathbf{2}$ \\
\hline Relaxed overstrength homogenity $(\mathbf{\Omega})$ & $\Omega_{\max }<\mathbf{1 , 5 0} \Omega_{\min }$ \\
criterion & $\mathbf{1 , 3}<\lambda<\mathbf{2}$ \\
\hline Relaxed overstrength homogenity $(\mathbf{\Omega})$ & $\Omega_{\max }<\mathbf{1 , 5 0} \Omega_{\min }$ \\
\& Relaxed slenderness $(\boldsymbol{\lambda})$ criterion & $\mathbf{1 , 3}<\lambda<\mathbf{2 , 5}$ \\
\hline
\end{tabular}

Table 3 Design approaches

Case studies are proposed by CTICM for different "border line" cases, taking into account only circular hollow sections, as this class of sections fulfills the best all the requirements.

It is considered here that the worst situation in seismic design is to have the weak brace at the first level and strong braces at all other levels. So that case studies are studied where

$$
\Omega_{1}=1,00 \text { and } \Omega_{\mathrm{i}}=1,50 \text { for } \mathrm{i}>1 .
$$

For all cases, theoretical cross-sections are considered (diameter and thickness) to obtain a targeted reduced slenderness of 2 for $\mathrm{N}$ bracings.

So far, only 4-level frames with $\mathrm{N}$ bracings have been analyzed. Numerical analyses have been performed with Straus7 software [13]. Figure 2 shows the numerical model of case study $4 \mathrm{~N}-\mathrm{CHS}$ case study. 


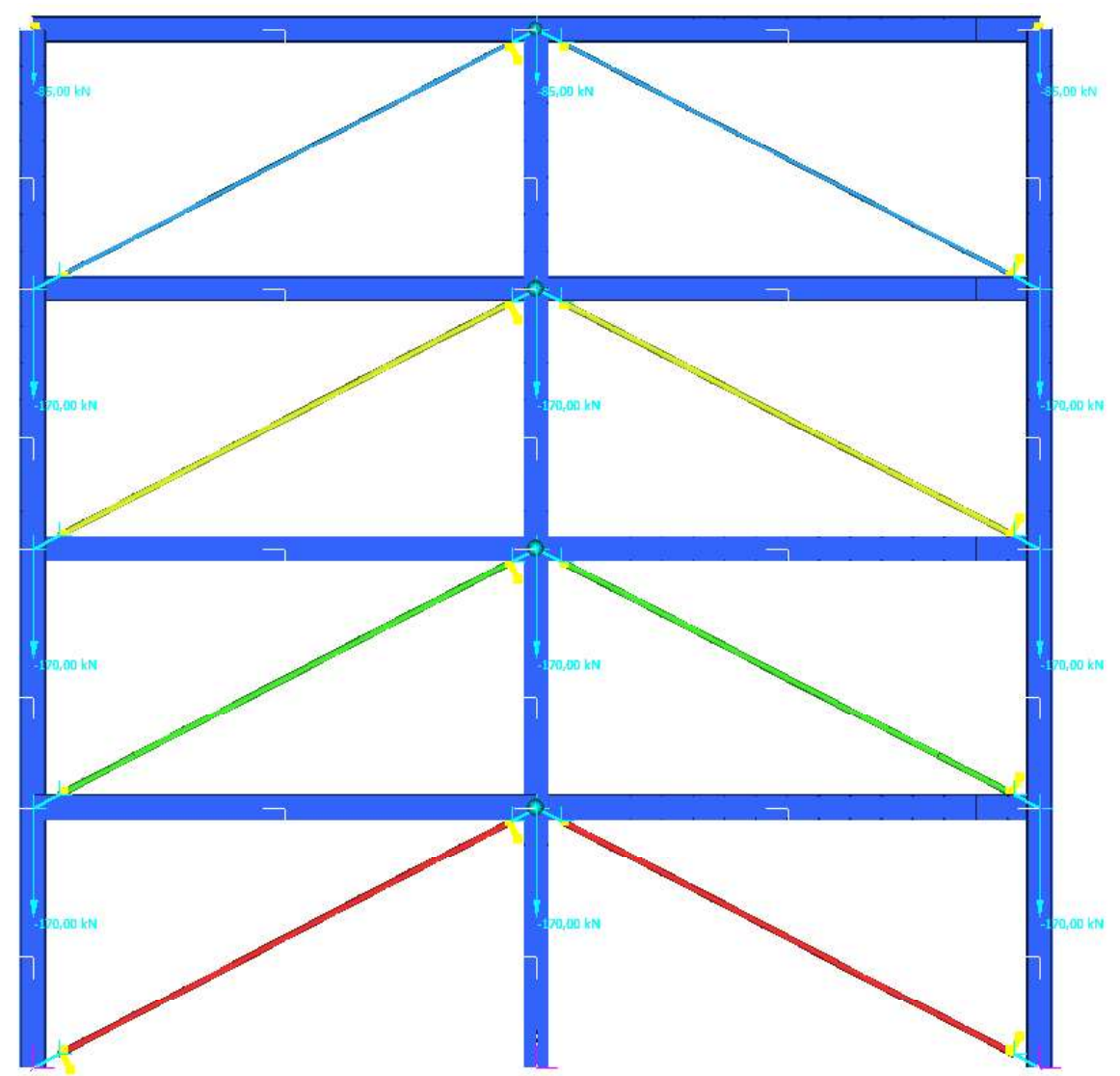

Figure 2 Analysis model of 4N-CHS case study

Three frames have been analyzed, which are designed according to the abovementioned principles (table 2). Bracings considered in different models can be seen in tables 4,5 and 6, which show that relaxation of the EC8 rules permits the adoption of lighter profiles.

$\begin{array}{ccccccccc}\text { Level } & \text { Section } & \text { Area } & \boldsymbol{\Omega} \mathbf{i} & \text { kbuckl } & \lambda \text { min } & \lambda \text { max } & I \text { min } & \text { Imax } \\ 1 & \text { CHS139.7*5.0 } & 21.2 & 2.09 & 0.90 & 1.47 & 1.47 & 481 & 481 \\ 2 & \text { CHS114.3*5.0 } & 17.2 & 2.07 & 0.90 & 1.81 & 1.81 & 257 & 257 \\ 3 & \text { CHS114.3*3.6 } & 12.5 & 2.00 & 0.90 & 1.79 & 1.79 & 192 & 192 \\ 4 & \text { CHS76.1*4.0 } & 9.1 & & 0.90 & & & 59 & 59\end{array}$

Table 4 4N-CHS EC8 design

$\begin{array}{cccccccc}\text { Level } & \text { NEd } & \text { Ad } & \mathbf{\Omega i} & \text { Dext } & \mathbf{t} & \text { Imin } & \text { Imax } \\ 1 & 229.0 & 8.3 & 1.00 & 101.7 & 2.7 & 102 & 102 \\ 2 & 197.8 & 10.8 & 1.50 & 102.5 & 3.5 & 132 & 132 \\ 3 & 140.0 & 7.6 & 1.50 & 101.5 & 2.5 & 94 & 94 \\ 4 & 70.8 & 3.4 & & 55.9 & 2.0 & 7 & 4155\end{array}$

Table 5 4N-CHS modified EC8 design with relaxed overstrength homogeneity

$\begin{array}{cccccccc}\text { Level } & \text { NEd } & \text { Ad } & \boldsymbol{\Omega i} & \text { Dext } & \mathbf{t} & \operatorname{Imin} & \text { Imax } \\ 1 & 229.0 & 8.3 & 1.00 & 82.6 & 3.3 & 65 & 65 \\ 2 & 197.8 & 10.8 & 1.50 & 83.5 & 4.3 & 85 & 85 \\ 3 & 140.0 & 7.6 & 1.50 & 82.3 & 3.1 & 60 & 60 \\ 4 & 70.8 & 3.4 & & 55.9 & 2.0 & 7 & 4155\end{array}$

Table 6 4N-CHS modified EC8 design with relaxed overstrength homogeneity \& relaxed slenderness 
Preliminary results show that with relaxed rules, maximum drifts obtained at each floor are far below the damage limits indicated by Eurocode 8 (figure 4). Although the general distribution of plasticity is quite homogeneous (figure 3), in some cases, there is an initiation of soft-storey floor, but since the seismicity is moderate, drift levels remain small, and do not seem to cause global problems.

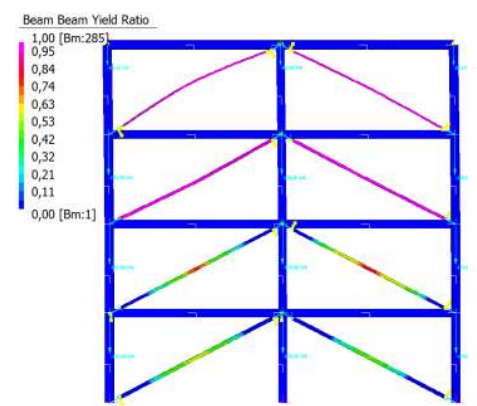

a. EC8 Design

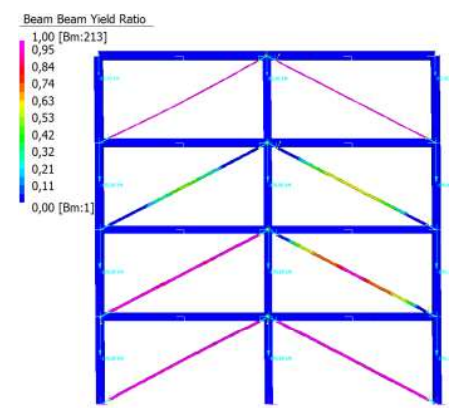

b. Relaxed omega

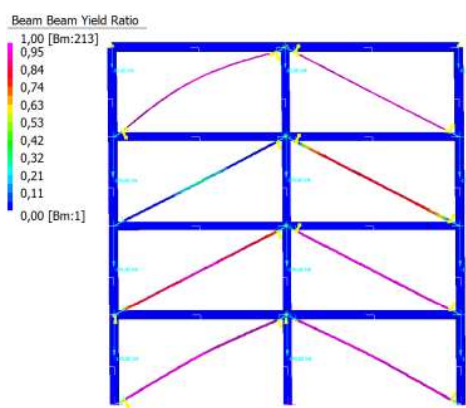

c. Relaxed omega and slenderness

Figure 3 Cumulative beam yield ratios and deformed shapes at the last step of accelerogram 1

It should also be noted that bracing sections are smaller in when the rules are relaxed, which means a saving in the steel amount used in the building.
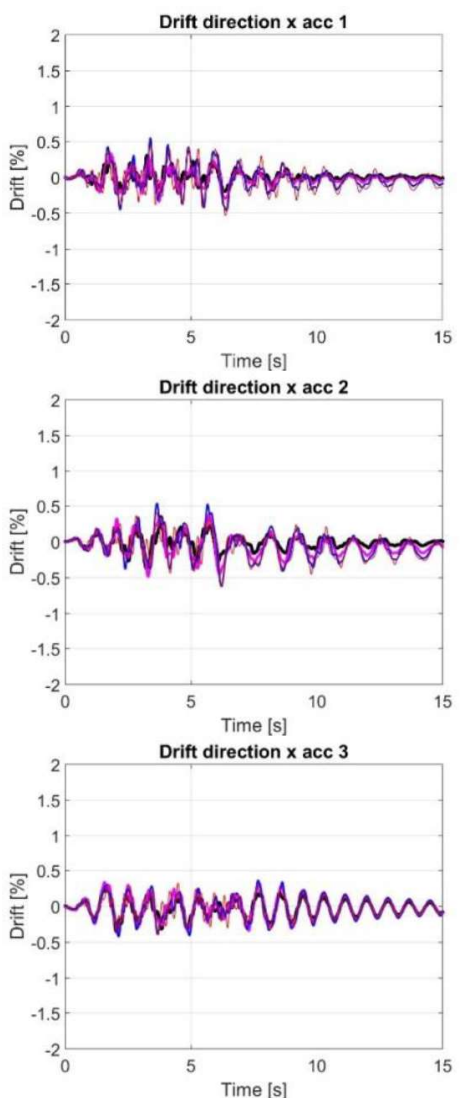

a. EC8 design
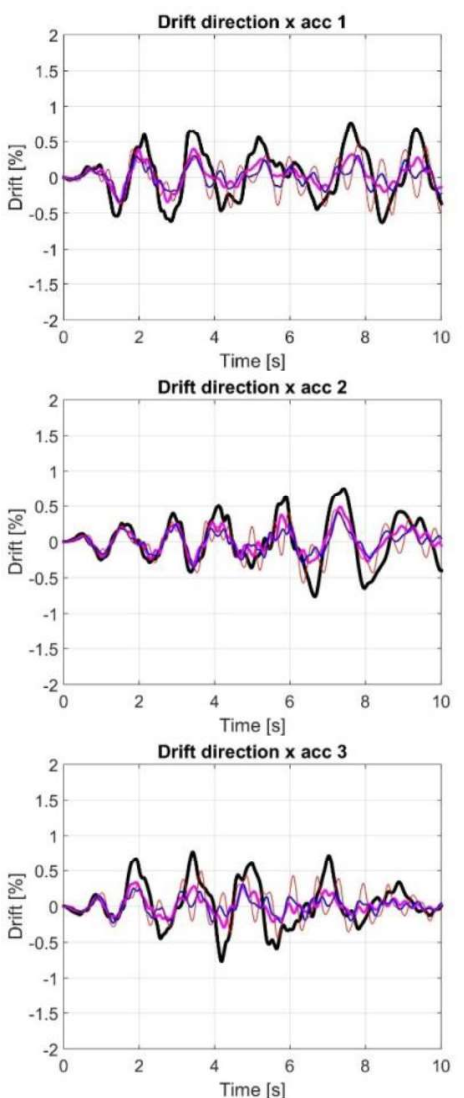

b. Relaxed omega
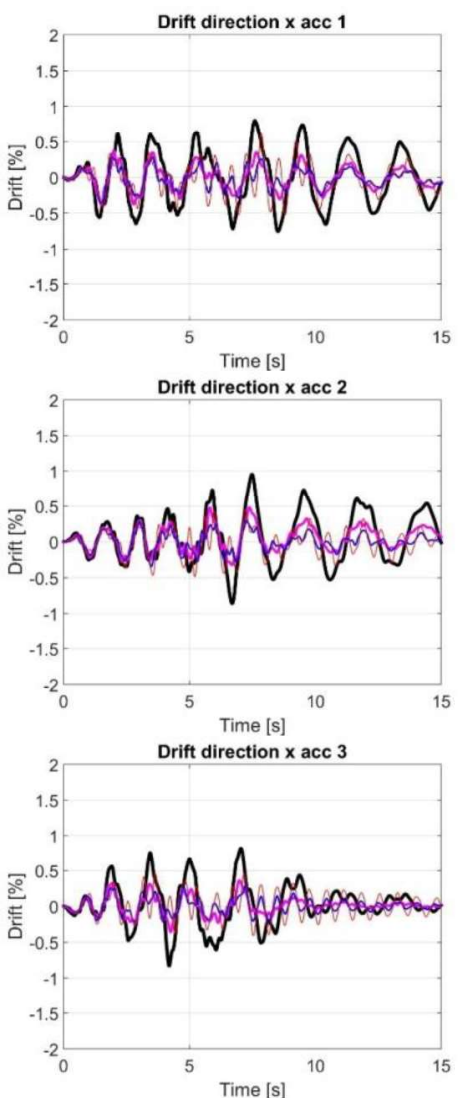

c. Relaxed omega and slenderness

—Floor 1 -Floor 2 -Floor 3 -Floor 4

Figure 4 Comparison between results in terms of floor drift ratios 


\section{CONCLUSIONS}

In this article, the outcomes of relaxing the stringent slenderness and overstrength homogenity criteria of Eurocode 8 have been investigated numerically. Nonlinear time history analyses have been performed on the case studies designed according to following design criteria:

i. $\quad$ EN 1998 overstrength homogeneity $\left(\Omega_{\max }<1,25 \Omega_{\min }\right)$ and slenderness limits $(1,3<\lambda<2)$

ii. Relaxed slenderness $(\lambda)$ rule $(1,3<\lambda<2,5)$

iii. Combination of relaxed slenderness $(\lambda)(1,3<\lambda<2,5)$ and overstrength homogenity $(\Omega)$ rules $\Omega_{\max }<1,50 \Omega_{\min }$

Case studies provided by project partner CTICM, are composed of 3 CBF structures, designed with abovementioned three design approaches, using linear static analysis with response spectrum approach. NLTHA have been performed under 3 artificial records, typical for moderate seismic zones. From the results of these analyses, preliminary observations have been made on the seismic performance of the frames designed according to different approaches.

This preliminary work showed that a certain amount of relaxation of slenderness and overstrength homogeneity rules permits designer to choose lighter profile sections from a larger profile database, though not worsening the seismic performance significantly. The research project is underway, and these investigations are currently being made with a broader range of case studies and acceleration time histories, and the results will be verified after experimental studies.

\section{ACKNOWLEDGMENT}

This study has been realized thanks to the research fund received from European commission with the contract MEAKADO RFSR-CT-2013-00022.

\section{REFERENCES}

[1] H. Degèe, C.A. Castiglioni, P.O. Martin, I. Calderon, B. Hoffmeister, Eight International Conference on Advances in Steel Structures

[2] Eurocode 8, Design of structures for earthquake resistance, 2003.

[3] P. Pinto, Design for Low-Moderate Seismic Risk, 2000

[4] K. Dominic and J. J. Zona, Design Tips for Steel in Low or Moderate Seismic Regions, 2006

[5] E.M. Hines and L.A. Fahnestock, Design Philosophy for Steel Structures in Moderate Seismic Regions (2010)

[6] E.M Hines, M. E. Appel and P. J. Cheever Collapse Performance of Low Ductility Chevron Braced Steel Frames in Moderate Seismic Regions, 2009

[7] A. Elghazouli, Assessment of European seismic design procedures for steel framed structures, 2008

[8] Gioncu and Mazzolani, Seismic Design of Steel Structures, 2014

[9] Murty and Malik, Challenges of Low-to-Moderate Seismicity in India, 2008

[10] A. Elghazouli, Seismic Design Procedures for Concentrically Braced Frames, 2003 
[11] A. Kanyilmaz, C.A. Castiglioni, Performance of Multi-level Composite Steel-Concrete Frames with Dissipative Devices, COMPDYN 2015, $5^{\text {th }}$ ECCOMAS Thematic Conference on Computational Methods in Structural Dynamics and Earthquake Engineering, Crete Island, Greece, 2015

[12] A. Kanyilmaz, Inelastic Cyclic numerical analysis of steel struts using distributed plasticity approach, COMPDYN 2015, 5th ECCOMAS Thematic Conference on Computational Methods in Structural Dynamics and Earthquake Engineering, Crete Island, Greece, 2015

[13] G+D Computing Pty Limited, Straus7. Theoretical Manual. Sydney (Australia), 2004. 